\title{
A new staging system for hepatocellular carcinoma associated with portal vein tumor thrombus
}

\author{
Wan Yee Lau ${ }^{1,2 \#}$, Kang Wang ${ }^{1 *}$, Xiu-Ping Zhang ${ }^{1,3 \#}$, Le-Qun Li $^{4}$, Tian-Fu Wen ${ }^{5}$, Min-Shan Chen ${ }^{6}$, \\ Wei-Dong Jia ${ }^{7,8}$, Li Xu ${ }^{6}$, Jie Shi ${ }^{1}$, Wei-Xing Guo ${ }^{1}$, Ju-Xian Sun ${ }^{1}$, Zhen-Hua Chen ${ }^{1}$, Lei Guo ${ }^{1}$, \\ Xu-Biao Wei ${ }^{1}$, Chong-De Lu ${ }^{1}$, Jie Xue ${ }^{1}$, Li-Ping Zhou ${ }^{1}$, Ya-Xing Zheng ${ }^{1}$, Meng Wang ${ }^{1}$, Meng-Chao Wu ${ }^{1}$, \\ Shu-Qun Cheng'
}

${ }^{1}$ Department of Hepatic Surgery VI, Eastern Hepatobiliary Surgery Hospital, The Second Military Medical University, Shanghai, China; ${ }^{2}$ Faculty of Medicine, The Chinese University of Hong Kong, Hong Kong, China; ${ }^{3}$ Faculty of Hepato-Pancreato-Biliary Surgery, The First Medical Center of Chinese People's Liberation Army (PLA) General Hospital, Beijing, China; ${ }^{4}$ Department of Hepatobiliary Surgery, Affiliated Tumor Hospital of Guangxi Medical University, Nanning, China; ${ }^{5}$ Department of Liver Surgery \& Transplantation Center, West China Hospital of Sichuan University, Chengdu, China; ${ }^{6}$ Department of Hepatobiliary Surgery, Sun Yat-sen University Cancer Center, Guangzhou, China; ${ }^{7}$ Department of General Surgery, Affiliated Provincial Hospital, Anhui Medical University, Hefei, China; ${ }^{8}$ Anhui Province Key Laboratory of Hepatopancreatobiliary Surgery, Hefei, China

Contributions: (I) Conception and design: WY Lau, K Wang, XP Zhang, SQ Cheng; (II) Administrative support: None; (III) Provision of study materials or patients: None; (IV) Collection and assembly of data: LQ Li, TF Wen, MS Chen, WD Jia, L Xu, J Shi, WX Guo, JX Sun, ZH Chen, L Guo, XB Wei, CD Lu, J Xue, LP Zhou, YX Zheng, M Wang, MC Wu; (V) Data analysis and interpretation: WY Lau, K Wang, XP Zhang, SQ Cheng; (VI) Manuscript writing: All authors; (VII) Final approval of manuscript: All authors.

\#These authors contributed equally to this work.

Correspondence to: Shu-Qun Cheng, MD, PhD. Eastern Hepatobiliary Surgery Hospital, Second Military Medical University, 225 Changhai Road, Shanghai 200433 China. Email: chengshuqun@aliyun.com.

Background: A new staging system for patients with hepatocellular carcinoma (HCC) associated with portal vein tumor thrombus (PVTT) was developed by incorporating the good points of the BCLC classification of HCC, and by improving on the currently existing classifications of HCC associated with PVTT.

Methods: Univariate and multivariate analysis with Wald $\chi^{2}$ test were used to determinate the clinical prognostic factors for overall survival (OS) in patients with HCC and PVTT in the training cohort. Then the conditional inference trees analysis was applied to establish a new staging system.

Results: A training cohort of 2,179 patients from the Eastern Hepatobiliary Surgery Hospital and a validation cohort of 1,550 patients from four major liver centers in China were enrolled into establishing and validating a new staging system. The system was established by incorporating liver function, general health status, tumor resectability, extrahepatic metastasis and extent of PVTT. This staging system had a good discriminatory ability to separate patients into different stages and substages. The median OS for the two cohorts were 57.1 (37.2-76.9), 12.1 (11.0-13.2), 5.7 (5.1-6.2), 4.0 (3.3-4.6) and 2.5 (1.7-3.3) months for the stages 0 to $I V$, respectively $(\mathrm{P}<0.001)$ in the training cohort. The corresponding figures for the validation cohort were 6.4 (4.9-7.9), 2.8 (1.3-4.4), 10.8 (9.3-12.4), and 1.5 (1.3-1.7) months for the stages II to $\mathrm{IV}$, respectively $(\mathrm{P}<0.001)$. The mean survival for stage 0 to 1 were $37.6(35.9-39.2)$ and 30.4 (27.4-33.4), respectively $(\mathrm{P}<0.001)$.

Conclusions: A new staging system was established which provided a good discriminatory ability to separate patients into different stages and substages after treatment. It can be used to supplement the other HCC staging systems.

Keywords: Hepatocellular carcinoma (HCC); portal vein tumor thrombus (PVTT); staging system; overall survival (OS) 
Submitted Nov 15, 2019. Accepted for publication Apr 28, 2020.

doi: $10.21037 /$ hbsn-19-810

View this article at: https://dx.doi.org/10.21037/hbsn-19-810

\section{Introduction}

Hepatocellular carcinoma (HCC) ranks as the sixth most common neoplasm and the third leading cause of cancer death (1). Portal vein tumor thrombosis is an important prognostic factor of long-term overall survival (2-4), occurring in $12.5 \%$ to $39.7 \%$ of patients with HCC at diagnosis, and up to $64.7 \%$ at autopsy. If left untreated, a median survival time (MST) of 2.7 to 4.0 months was reported $(5,6)$. It has been considered as the bottleneck in the treatment of HCC (7).

Several clinical classifications of HCC has been proposed. The Barcelona Clinic Liver Cancer (BCLC) system is most commonly used (8), and is endorsed by the EASL-EORTC GP guidelines (9-11). However, the BCLC staging system classifies all patients with HCC associated with PVTT into stage $\mathrm{C}$ and recommends sorafenib as the only treatment $(12,13)$. Such a classification is too rough and refinement is needed. A refinement can help clinicians and patients to appreciate the extent of HCC, to guide treatment, to predict prognosis, to compare treatment results and diagnostic accuracies of the various options at a comparable tumor staging.

There are currently two available systems to classify the extent of HCC associated with PVTT: the Cheng's Classification for PVTT (Type I-IV) and the Japanese staging system (Vp1-Vp4) (14-16). The Cheng's Classification comprises of 4 categories $(14,15)$ (Type I, II, III, IV) based on the extent of PVTT invasion into the portal vein. This classification is very similar to the Japanese classification with the exception that it combines $\mathrm{Vp} 1$ and $\mathrm{Vp} 2$ as Type I, and it subdivides Vp4 into Type III and IV (17). However, these two systems do not have the key prognostic predictors which include liver function, resectability of tumor, overall health status and recommended treatment modalities. Thus, a new staging system which incorporates these factors and recommends treatment options in different stages of HCC associated with PVTT is needed.

This study aimed to set up a new staging system by incorporating liver function, resectability of tumor, extent of PVTT, overall health status and extrahepatic metastasis. Patients with HCC associated with PVTT were separated into different stages and substages, and their long-term overall survival (OS) outcomes were analyzed. We present the following article in accordance with the TRIPOD reporting checklist (available at https://hbsn.amegroups.com/article/ view/10.21037/hbsn-19-810/rc).

\section{Methods}

\section{Patients and study design}

The demographic, clinical and pathological data of consecutive patients with HCC associated with PVTT who were treated in five centers in China were retrospectively reviewed. The data from the Eastern Hepatobiliary Surgery Hospital in Shanghai from January 2002 to January 2017 were used as a training cohort to establish the new staging system. Data on patients who were treated in four other major liver centers in China from 2012 to 2017 were used as a validation cohort to assess the performance of this newly established system. The names of the four participating hospitals are Affiliated Tumor Hospital of Guangxi Medical University, West China Hospital of Sichuan University, Sun Yat-sen University Cancer Center and Affiliated Provincial Hospital of Anhui Medical University. The study was conducted in accordance with the Declaration of Helsinki (as revised in 2013). The study was approved by the Ethics Committees of all the five hospitals (Permit Number: EHBHKY-2019-001-017). Informed consent was obtained from all the patients prior to treatment. All the patients who were regularly followed-up as we have described previously (18). All the patients who entered into this study were re-examined in their hospital within a month after surgery. Follow-up was performed on the outpatient bases and/or by telephone calls at 1 to 3 -month intervals according to the standard epidemiologic procedure. During follow-up, serum levels of alpha-fetoprotein (AFP) and HBV DNA, liver function, and ultrasonic examination of the liver were carried out. For patients who were suspected to develop recurrence or progressive diseases, abdominal computed tomography and/ or magnetic resonance imaging were used to confirm the diagnosis. RFA, PEI, TACE or multi-disciplinary treatment (MDT) was selected for treatment of HCC recurrence according to the liver function, location and number 
of recurrence nodules, and intra- and/or extra-hepatic metastases.

Extrahepatic metastasis was defined as multiple extrahepatic metastases in a single organ, or extrahepatic metastases in multiple organs. Tumor resectability was defined as a single tumor or multiple tumors which was (were) confined to a single hemiliver, a sector or a segment, and the volume of the liver remnant after liver resection was predicted to be more than $30 \%$ (19).The patients enrolled into this study all died of HCC progression or recurrence. Death due to other causes were excluded from this study. Patients who had bile duct and hepatic venous tumor thrombus were excluded. The median survival time was used for the training cohort, while the median or mean time was used for the validation cohort due to limited follow-up time for the patients (mean time for stage 0 to I and median time for stage II to IV, respectively).

\section{Treatments of patients}

All patients who entered into this study were reviewed at the multi-disciplinary HCC boards of the respective hospitals when the diagnosis of HCC was first made. Information on the therapeutic risks and benefits was provided to the patients. A shared-decision was made between the clinicians and the patients. All patients who had adequate liver function and radiologically resectable tumor were initially evaluated for partial hepatectomy. If the patient was not a surgical candidate, transarterial chemoembolization (TACE), TACE combined with sorafenib (TACE-Sor), TACE combined with external radiotherapy (TACE-RT), sorafenib or local ablative procedures such as percutaneous ethanol injection or radiofrequency ablation were offered, depending on the size, number and position of the tumor. Patients with advanced diseases were offered systemic therapy. The treatment was performed under standard procedures as previously described (20). A written informed consent was obtained from all the patients.

\section{Statistical analysis}

Survival was analyzed by the Kaplan-Meier method and survival curves were compared by the log-rank test. The overall survival (OS) was defined as the time from the date of first diagnosis of HCC associated with PVTT to death, the last follow-up or the date of data censoring (1st Jan. 2017). To check the similarity between the Training Cohort and the Validation Cohort, the demographic, clinical, and laboratory characteristics of patients on presentation in the two sets were compared using the Pearson's chi-square test for categorical variables, and the Wilcoxon rank sum test for continuous variables. Categorical variables were shown as number (percentage). Univariate and multivariate cox regression was used to identify significant variables related with OS. Partial Wald $\chi^{2}$ test (21) and conditional inference trees (22) were used to determinate the last variables to establish the new staging system. The Schoenfeld residual plots of each prognostic factors were observed and transformed into the time-dependent relative coefficients. A value of $\mathrm{P}<0.05$ was statistically significant. The analysis was performed with the SPSS software (IBM SPSS Statistics, USA, version 24.0) and the R-project (R Foundation for Statistical Computing, Vienna, Austria, version 3.4.4).

\section{Results}

\section{Patient characteristics}

Of the 3,729 patients with HCC associated with PVTT in this study, 2,179 patients formed the training cohort and 1550 patients the validation cohort. The characteristics of the patients in the two cohorts are shown in Table 1. There were no significant differences in age, albumin, HBsAg, and AFP level between the two cohorts. In the Training Cohort, there were significantly more patients with abnormal ALT levels, cirrhosis and tumor size $>5 \mathrm{~cm}$ than the Validation Cohort $(52.7 \%$ vs. $41.9 \%, \mathrm{P}<0.001 ; 70.5 \%$ vs. $61.5 \%$, $\mathrm{P}<0.001$ and $80.3 \%$ vs. $74.9 \%, \mathrm{P}<0.001$, respectively). There were also significantly less patients with multiple tumors than in the validation cohort $(25.1 \%$ vs. $51.5 \%$, $\mathrm{P}<0.001)$. The characteristics of all the patients who were enrolled into this study are shown in Table S1.

\section{Patients treatments}

The first treatments are shown in Table 2. In the training cohorts, there were 1,067 (49.1\%) patients who underwent liver resection, 706 (32.4\%) TACE, 34 (1.6\%) molecular targeting drugs or chemotherapy, 177 (8.1\%) TACE-SUR, 107 (4.9\%) RT-TACE and 88 (4.0\%) the best supportive care (BSC), respectively. The median OS time was 17.6 (95\% CI: 15.7-19.6) for liver resection, 3.7 (CI: 3.4-4.0) for TACE, 5.6 (CI: 5.4-5.9) for MDT or Chemotherapy, 8.1 (CI: 7.3-9.0) for TACE-SUR, 9.5 (CI: 6.8-12.3) for RT-TACE and 2.5 (CI: 1.7-3.3) for BSC, respectively in the Training Cohort. In the validation cohort, there were 
Table 1 Patient's characteristics of all the enrolled patients

\begin{tabular}{|c|c|c|c|}
\hline Variables & Training cohort $(n=2,179)$ & Validation cohort $(n=1,550)$ & $\mathrm{P}$ \\
\hline Male & $1,961(90.4)$ & 1,362 (87.9) & 0.015 \\
\hline Female & $210(9.6)$ & $188(12.1)$ & \\
\hline Age, years, n (\%) & & & 0.95 \\
\hline$>50$ & $1,051(48.2)$ & $746(48.1)$ & \\
\hline \multicolumn{4}{|l|}{ Biochemistries, n (\%) } \\
\hline Total bilirubin (mmol/L) & & & 0.001 \\
\hline$\leq 18.8$ & $1,374(63.1)$ & $1,074(69.3)$ & \\
\hline$\leq 34$ & $295(13.5)$ & $198(12.8)$ & \\
\hline$>34$ & $1,884(86.5)$ & 1,352 (87.2) & \\
\hline ALT $(\mu \mathrm{L} / \mathrm{L})$ & & & $<0.001$ \\
\hline$\leq 44$ & $1,031(47.3)$ & $901(58.1)$ & \\
\hline$>44$ & $1,148(52.7)$ & $649(41.9)$ & \\
\hline $\mathrm{HbsAg}$ & & & 0.447 \\
\hline Positive & $1,908(87.6)$ & $1,370(88.4)$ & \\
\hline Negative & $271(12.4)$ & $180(11.6)$ & \\
\hline AFP (ng/mL) & & & 0.071 \\
\hline$\leq 400$ & $851(39.1)$ & $651(42.0)$ & \\
\hline$>400$ & $1,328(60.9)$ & $899(58.0)$ & \\
\hline Tumor size $(\mathrm{cm})$ & & & $<0.001$ \\
\hline$\leq 5$ & $429(19.7)$ & $389(25.1)$ & \\
\hline$>5$ & $1,750(80.3)$ & $1,161(74.9)$ & \\
\hline Tumor number & & & $<0.001$ \\
\hline Single & $1,632(74.9)$ & $752(48.5)$ & \\
\hline Multiple & $547(25.1)$ & $798(51.5)$ & \\
\hline Local tumor resectability & & & $<0.001$ \\
\hline Yes & $1,067(49.0)$ & $1,054(68.0)$ & \\
\hline No & $1,112(51.0)$ & 496 (32.0) & \\
\hline
\end{tabular}

Table 1 (continued) 
Table 1 (continued)

\begin{tabular}{|c|c|c|c|}
\hline Variables & Training cohort $(n=2,179)$ & Validation cohort $(n=1,550)$ & $P$ \\
\hline No & $1,895(87.0)$ & $1,387(89.5)$ & \\
\hline Yes & $284(13.0)$ & $163(10.5)$ & \\
\hline Cheng's classification for PVTT, n (\%) & & & $<0.001$ \\
\hline I & $319(14.6)$ & $276(17.8)$ & \\
\hline II & $716(32.9)$ & $249(16.1)$ & \\
\hline III & $647(29.7)$ & $216(13.9)$ & \\
\hline IV & $175(8.0)$ & $24(1.5)$ & \\
\hline 1 and 2 & $319(14.6)$ & $276(17.8)$ & \\
\hline 3 & $716(32.9)$ & $249(16.1)$ & \\
\hline 4 & $822(37.7)$ & $240(15.4)$ & \\
\hline EOCG-PS, n (\%) & & & 0.070 \\
\hline 0 & $1,067(49.0)$ & $785(50.6)$ & \\
\hline $1-2$ & $1,024(47.0)$ & $683(44.1)$ & \\
\hline $3-4$ & $88(4.0)$ & $82(5.3)$ & \\
\hline Child-Pugh, n (\%) & & & 0.071 \\
\hline
\end{tabular}

PVTT, portal vein tumor thrombus.

$1,054(68.0 \%)$ patients who underwent liver resection, 249 (16.1\%) TACE, 24 (1.5\%) molecular targeting drugs or chemotherapy, 132 (8.5\%) TAI, 5 (4.9\%) RFA, and 82 (5.3\%) BSC, respectively. The mean OS time was 35.8 (CI: 34.3-37.3) for liver resection, the median OS time was 4.0 (CI: 2.9-5.1) for TACE, 2.5 (CI: 1.1-3.9) for MDT or chemotherapy, 7.7 (CI: 6.3-9.1) for TAI, 4.0 (CI: 0-10.4) for RF and 1.5 (CI: 1.3-1.7) for BSC, respectively (Table S2).

\section{Importance of the clinical prognostic factors}

Through univariate and multivariate cox regression analyses, the extent of PVTT (HR with 95\% CI: 3.023 ; 2.537-3.602), the Eastern Cooperative Oncology Group performance status $0-1$ (ECOG PS) (3.451; 2.919-4.082) and $8.703 ; 6.813-11.118)$, the Pugh-Child grading (8.703;
6.813-11.118), extrahepatic metastasis (1.711; 1.475-1.985), local tumor resectability $(1.312 ; 1.163-1.481)$ and whether the main PV was involved by PVTT $(1.599 ; 1.443-1.773)$ were independently associated with OS (Figure $1 \mathrm{~A}, 1 \mathrm{~B}$ ). As patients with Child-Pugh $\mathrm{C}$ were in tune with patients with ECOG PS 3-4, these two models were respectively established in the multivariate analysis. For further analysis, the above six indicators with a high Wald $\chi^{2}$ value [209.6 (299.9), 299.9, 152.8, 79.8, 50.3 and 19.5] were filled into the process of the conditional inference trees by the permutation tests in Figure 2A,2B. Table S3 shows the conditions of each variable in each node by the conditional inference trees. The scaled Schoenfeld residual plots showed that the coefficient estimates varied early in time and quickly stabilized by approximately two months. The pooled two months coefficients were obtained and 
Table 2 Number of patients who underwent the first treatment in training cohort and validation cohort

\begin{tabular}{|c|c|c|c|c|c|c|c|c|}
\hline $\begin{array}{l}\text { The new staging } \\
\text { system }\end{array}$ & \multicolumn{8}{|c|}{ First treatment, n (\%) } \\
\hline $\begin{array}{l}\text { Training cohort } \\
(n=2,179)\end{array}$ & $1,067(49.1)$ & $706(32.4)$ & $34(1.6)$ & $177(8.1)$ & $107(4.9)$ & & & $88(4.0)$ \\
\hline 0 & $322(14.8)$ & 0 & 0 & 0 & 0 & 0 & 0 & 0 \\
\hline III & 0 & $120(5.5)$ & $34(1.6)$ & 77 (3.5) & 0 & 0 & 0 & 0 \\
\hline IV & 0 & 0 & 0 & 0 & 0 & 0 & 0 & $88(4.0)$ \\
\hline $\begin{array}{l}\text { Validation cohort } \\
(n=1,550)\end{array}$ & $1,054(68.0)$ & $249(16.1)$ & $24(1.5)$ & & & $132(8.5)$ & $5(0.4)$ & $82(5.3)$ \\
\hline II & 0 & $182(11.7)$ & $12(0.8)$ & 0 & 0 & $90(5.8)$ & $4(0.3)$ & 0 \\
\hline III & 0 & $67(4.3)$ & $12(0.8)$ & 0 & 0 & $42(2.7)$ & $1(0.1)$ & 0 \\
\hline IV & 0 & 0 & 0 & 0 & 0 & 0 & 0 & $82(5.3)$ \\
\hline
\end{tabular}

MDT, multimodality treatment; Che, systemic chemotherapy; Sor, sorafenib; RT, radiotherapy; TAl, transhepatic arterial infusion; RF, radiofrequency ablation; BSC, best supportive care; TACE, transcatheter arterial chemoembolization.

transformed into the relative coefficient for use in this study. The reference category of each prognostic factor was assigned a value of zero (Table S4).

\section{Establishment of the new staging system}

Figure 3 shows the new staging system for patients with HCC associated with PVTT. In brief, patients with either poor general performance function (ECOG PS 3-4) or decompensated liver function (Pugh-Child grade C) were classified into stage IV (the terminal stage). The presence of extrahepatic metastasis was used to stratify patients into stage III (the advanced stage). The remaining patients were stratified into stage II with unresectable HCC (the intermediate stage) and stage 1 with resectable HCC (the early stage). Patients with microvascular invasion diagnosed on microscopic examination for resected specimens obtained after liver resection were stratified as stage 0 (the very early stage). Patients in stages I to III were further classified into $\mathrm{A}$ (without main portal vein invasion) and $\mathrm{B}$ (with main portal vein invasion).

\section{Validation of the new staging system}

The survival profiles of the patients for the new staging system are shown in Table S5 and Figure 4. The new staging system stratified patients well into distinct groups and sub-groups. There were significantly less patients who were classified into the new classification stage $0(\mathrm{P}<0.001)$ in the training cohort than the validation cohort. In the training cohort, the median survival for stage 0 to IV were 57.1 (37.2-76.9), 12.1 (11.0-13.2), 5.7 (5.1-6.2), $4.0(3.3-4.6)$ and $2.5(1.7-3.3)$ months for the stages 0 to IV, respectively $(\mathrm{P}<0.001)$. The cumulative $1-, 3$ - and 5 -year OS rates were $86.3 \%, 69.7 \%$ and $61.9 \%$ for stage 0 patients, $50.2 \%, 28.9 \%$ and $19.3 \%$ for stage I, $26.8 \%$, $8.7 \%$ and $2.9 \%$ for stage II, $10.6 \%, 0 \%, 0 \%$ for stage III, respectively, $\mathrm{P}<0.001$. For patients in stage IV, the 1 -year survival rate was $0 \%$ (Figure 4). In the Validation Cohort, the mean survival for stage 0 to I were 37.6 (35.9-39.2) and 30.4 (27.4-33.4), respectively $(\mathrm{P}<0.001)$. The median survival for stage II to IV were 6.4 (4.9-7.9), 2.8 (1.3-4.4), 10.8 (9.3-12.4), and $1.5(1.3-1.7)$ months, respectively 
A Unitvariate analysis

\begin{tabular}{|c|c|c|c|c|}
\hline $\begin{array}{l}\text { Variables } \\
\text { PS } 0\end{array}$ & Hazard Ratio(95\%Cl) & $\mathrm{HR}(95 \% \mathrm{Cl})$ & P-value & Wald $x^{2}$ \\
\hline PS 1-2 & $\rightarrow$ & $3.339(3.018-3.694)$ & $<0.001$ & 547.764 \\
\hline PS 3-4 & & $8.995(7.141-11.331)$ & $<0.001$ & 347.972 \\
\hline Tumor resectability,yes vs no & $\rightarrow$ & $3.101(2.811-3.421)$ & $<0.001$ & 509.994 \\
\hline PVTT,yes vs no & $\longrightarrow$ & $4.958(4.217-5.829)$ & $<0.001$ & 375.820 \\
\hline Extrahepatic metastasis,yes vs no & -6 & $3.596(3.138-4.120)$ & $<0.001$ & 338.989 \\
\hline MPV,yes vs no & 量 & $2.310(2.094-2.548)$ & $<0.001$ & 278.728 \\
\hline Child-pugh, C vs A-B & $\longrightarrow$ & $4.574(3.669-5.701)$ & $<0.001$ & 182.988 \\
\hline Tumor size $(>5 \mathrm{vs}<=5 \mathrm{cmcm}$ ) & $\square$ & $1.324(1.198-1.463)$ & $<0.001$ & 30.197 \\
\hline Albumin (>34 vs $<=34 \mathrm{~g} /$ ) & $=$ & $1.477(1.267-1.722)$ & $<0.001$ & 24.808 \\
\hline Total bilirubin (>18.8 vs $<=18.8 \mathrm{mmol} / \mathrm{L}$ ) & $\square$ & $1.278(1.154-1.415)$ & $<0.001$ & 22.347 \\
\hline Cirrhosis, Y vs N & $\square$ & $1.271(1.140-1.418)$ & $<0.001$ & 18.581 \\
\hline AFP (>400 vs $<=400 \mathrm{ng} / \mathrm{mL}$ ) & $\square$ & $1.250(1.129-1.383)$ & $<0.001$ & 18.536 \\
\hline ALT (>44 vs $<=44 \mathrm{u}$ I/1) & & $1.115(1.012-1.229)$ & 0.028 & 4.809 \\
\hline Tumor number,M vs S & 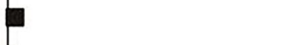 & $1.119(1.295-0.968)$ & 0.129 & 2.305 \\
\hline $\mathrm{HbsAg}, \mathrm{P}$ vs N & & $1.034(0.893-1.197)$ & 0.655 & 0.199 \\
\hline Age, $<=50$ vs $>50$ years & & $1.017(0.923-1.121)$ & 0.731 & 0.118 \\
\hline Gender, M vsF & 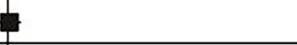 & $1.026(0.870-1.209)$ & 0.762 & 0.092 \\
\hline
\end{tabular}

B Multivariate analysis

\begin{tabular}{|c|c|c|c|c|}
\hline Variables & Hazard Ratio $(95 \% \mathrm{Cl})$ & $\mathrm{HR}(95 \% \mathrm{Cl})$ & P-value & Wald $x^{2}$ \\
\hline PS 0 & & & & \\
\hline PS 1-2 & & $3.451(2.919-4.082)$ & $<0.001$ & 209.625 \\
\hline PS 3-4 & & $8.703(6.813-11.118)$ & $<0.001$ & 299.924 \\
\hline \#Child-pugh,C vs A-B & & $8.703(6.813-11.118)$ & $<0.001$ & 299.924 \\
\hline PVTT,yes vs no & & $3.023(2.537-3.602)$ & $<0.001$ & 152.812 \\
\hline MPV,yes vs no & & $1.599(1.443-1.773)$ & $<0.001$ & 79.759 \\
\hline Extrahepatic metastasis,yes vs no & & $1.711(1.475-1.985)$ & $<0.001$ & 50.274 \\
\hline Tumor resectability,yes vs no & & $1.312(1.163-1.481)$ & $<0.001$ & 19.452 \\
\hline
\end{tabular}

Figure 1 Univariate (A) and multivariate analysis (B) with Wald $\chi^{2}$ test for OS in the patients with hepatocellular carcinoma associated with portal vein tumor thrombus. Two models were established by serval variables with or without the Child-Pugh.

$(\mathrm{P}<0.001)$. The cumulative 1-, 3 - and 5-year OS rates were $78.1 \%, 67.5 \%$ and $61.4 \%$ for stage $0,65.6 \%, 56.2 \%$ and $48.6 \%$ for stage I, $29.6 \%, 18.7 \%$ and $12.8 \%$ for stage II, $12.5 \%, 0 \%, 0 \%$ for stage III, $1.2 \%, 0 \%, 0 \%$ for stage IV, respectively $(\mathrm{P}<0.001)$ (Figure $4 C)$. The survival analyses for patients in the new staging system are shown in Figure $4 B, 4 D$. The patients were also well stratified into the distinct sub-groups $(\mathrm{P}<0.005)$.

\section{Comparison of predictive accuracy and clinical usefulness of the new staging system to other common staging systems}

In comparison to other commonly used staging systems, the time-dependent-ROC curve area analysis was used to determine which staging systems were good at predicting the OS. As shown in Figure $5 A, 5 B$, the predicting capacity of the new staging system was better than any of the other staging systems, including the CLIP, Okuda, BCLC, TNM, JIS, 
A

Conditional inference trees excluding the variable (Child-Pugh)

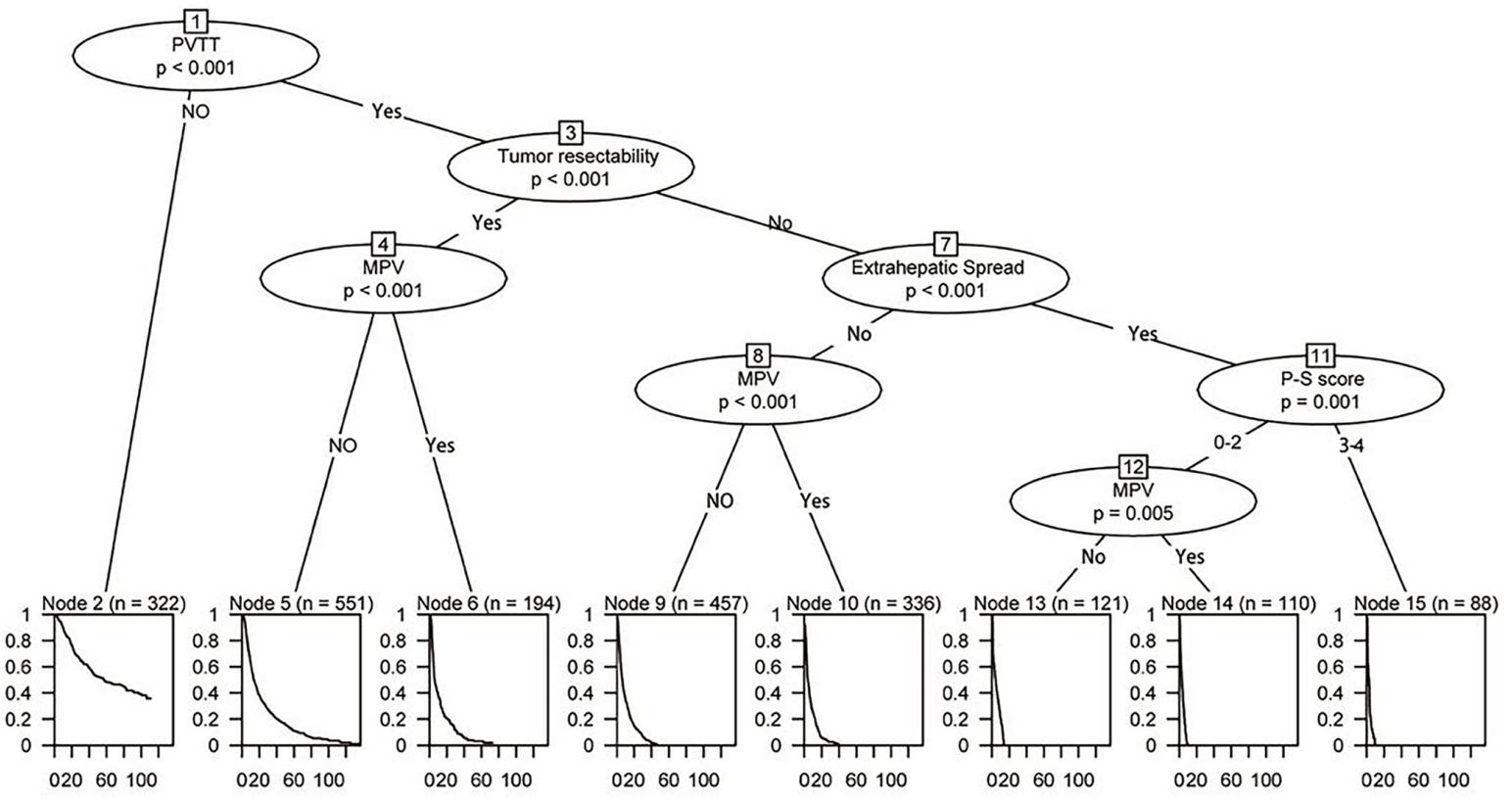

B Conditional inference trees including the variable (Child-Pugh)

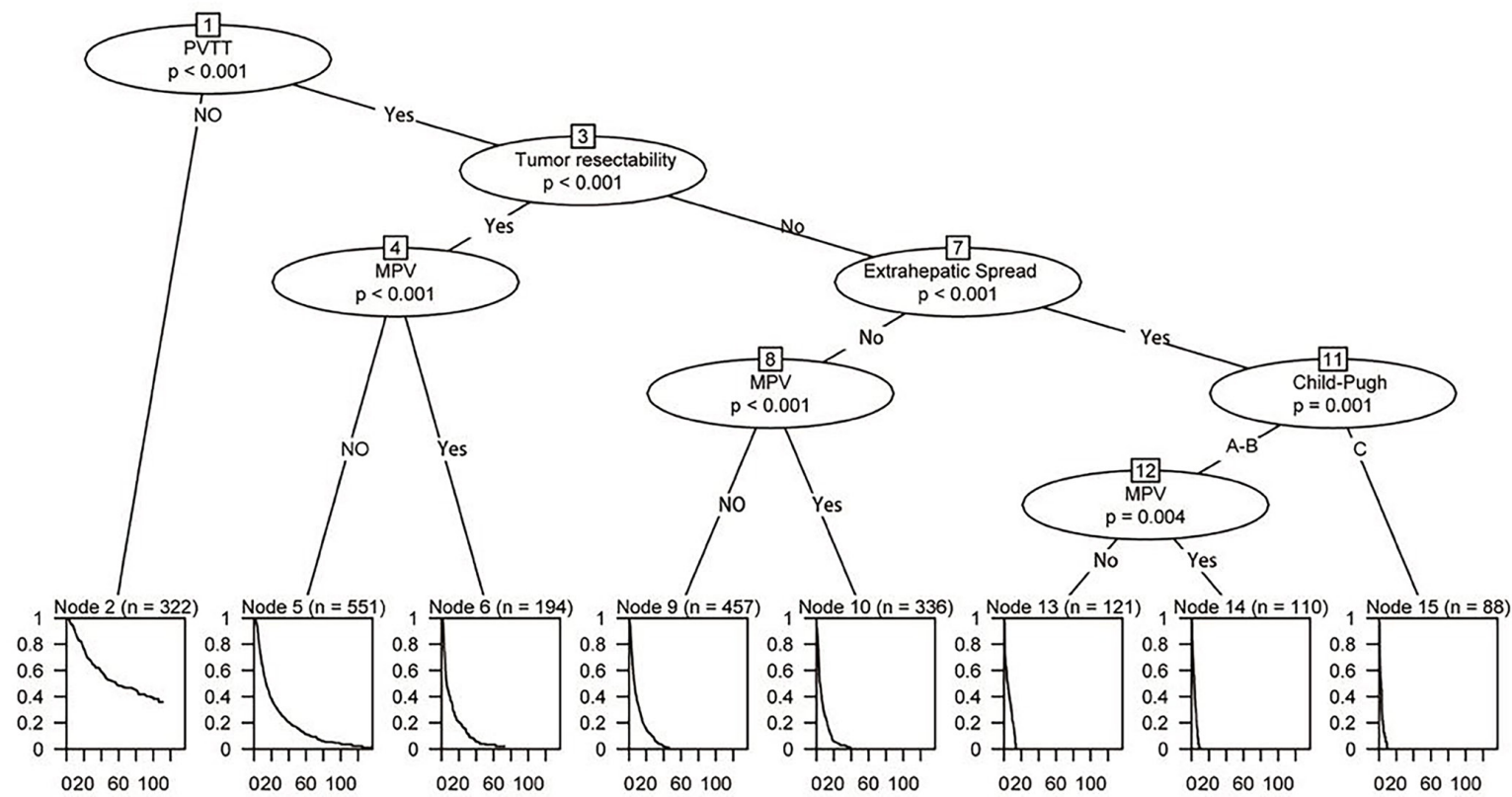

Figure 2 Conditional inference trees by serval variables without (A) or with (B) the Child-Pugh for overall survival in the patients with hepatocellular carcinoma associated with portal vein tumor thrombus.

CUPI, and Child-Pugh staging systems both in the training cohort and validation cohort. In addition, the decision curve analysis (DCA) was used to facilitate the comparison of clinical usefulness between the new staging system and the other staging systems. As shown in Figure 5C,5D, DCA showed that the new staging system provided superior net benefit when compared to those of the other staging systems both in the training cohort and validation cohort. 


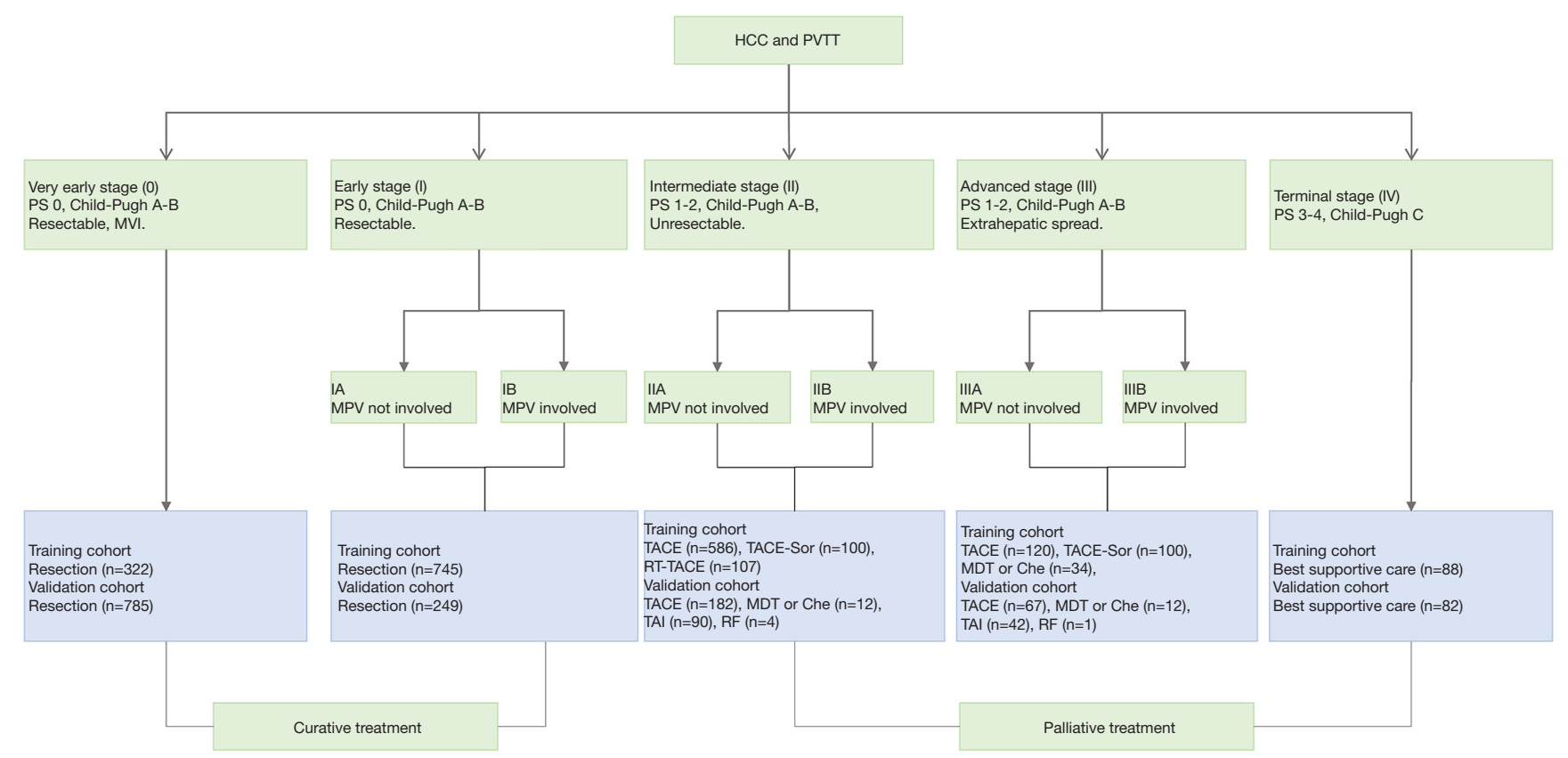


$\mathrm{RT}$, radiotherapy; TAI, transhepatic arterial infusion; RF, radiofrequency ablation; BSC, best supportive care; TACE, transcatheter arterial chemoembolization.

Figure 3 The new staging system for hepatocellular carcinoma associated with portal vein tumor thrombus.

\section{Discussion}

PVTT has been considered as a bottleneck in the treatment of HCC, and effective treatment of PVTT may improve treatment results for HCC (7). This study established a staging system in conjunction with a treatment algorithm which is applicable to patients with HCC associated with PVTT. It improves on the stage $\mathrm{C}$ classification of the widely accepted BCLC staging for HCC, and the Cheng's and the Japanese Classifications for HCC associated with PVTT, by incorporating liver function, general health status, tumor resectability, extrahepatic metastasis and extent of PVTT. This new staging system divides patients into five stages and with three substages for stages I, II and III. In the validation cohort which had significantly different patient characteristics, it still had good discriminatory power in stratifying patients with HCC associated with PVTT into different prognostic groups. This staging system may improve management of patients with HCC associated with PVTT by guiding clinical studies to compare the effectiveness of different treatment options for the different stages of disease.

The most common used HCC classification and scoring systems are the tumor-node-metastasis (TNM) staging, Okuda staging, Cancer of the Liver Italian Program (CLIP) scoring system, Barcelona Clinic Liver Cancer (BCLC) staging, French, Chinese University Prognostic Index (CUPI), Japanese Integrated Scoring (JIS), and Tokyo scoring system. The TNM system is based only on tumor characteristics and extent of invasion. It does not include hepatic function (23). According to the TNM staging, HCC patients with PVTT are defined as T3b, which could not be used to predict prognosis or survival outcomes of these patients. The BCLC system stratifies HCC patients into five categories ( 0 , very-early stage; $\mathrm{A}$, early-stage; $\mathrm{B}$, intermediate-stage; $\mathrm{C}$, advanced-stage; or $\mathrm{D}$, endstage disease) using tumor-related parameters (tumor size, number of nodules, vascular invasion, and extrahepatic spread) and patient characteristics, including Child-Pugh liver function class and performance status (9). According to the BCLC staging, HCC patients with PVTT are defined as BCLC C stage. The only proposed treatment option for this group of patients is sorafenib. The CLIP scoring system combines four tumor-related features-tumor extent and morphological features, serum alpha-fetoprotein levels, and portal vein thrombosis, with a cirrhosis severity index and the Child-Pugh score, to stratify HCC patients into groups (24). According to the CLIP system, PVTT is an independent risk factor to stratify HCC patients. The CUPI 



Figure 4 Overall survival of the different stagings and substagings for the new staging system for hepatocellular carcinoma associated with portal vein tumor thrombosis. (A,B) Training cohort; (C,D) validation cohort.

staging combines the conventional TNM system with factors relating to liver function and tumor load (25). The six prognostic factors are: the TNM stage, asymptomatic disease at presentation, total bilirubin level, ascites, alkaline phosphatase level, and alfa-fetoprotein level. The serological variables, liver function variables and PVTT (T3b of TNM) are combined to predict prognosis of HCC patients with PVTT. The JIS is based on the modified TNM system by the Liver Cancer Study Group of Japan and the ChildPugh score (26). Vascular invasion (PVTT) is acknowledged a staging index to stratify HCC patients. The CLIP, CUPI and JIS staging systems include PVTT as a staging index, and can therefore distinguish different prognosis of HCC patients with PVTT. However, all these scoring and staging systems have limitations because they could not be used to make a preoperative decision on liver resection (LR) for HCC patients with PVTT. This new PVTT system was established to provide a good discriminatory ability to separate patients into different stages and substages with treatments. It can be used to supplement the other HCC staging systems.

This new staging system divides treatment into: potentially curative treatment for patients in stage 0 and I, and palliative treatment for patients in stage II to IV. With this system, new and promising treatments can be compared stage-by-stage with the conventional treatment. The data from this study showed that in selected patients, long-term survival can be achieved with liver resection, and some patients with more advanced HCC can still derive substantial survival benefits from multi-modality treatments.

There is still no worldwide consensus on the management of patients with HCC associated with PVTT (27). Sorafenib is the only recommended treatment by BCLC for these patients. However, the reported median survival time is only 6.5 months in the Asian-Pacific study (28) and 10.5 months in the SHARP study (29). With recent advances, there have been increasing attempts to use more aggressive multimodality treatments for advanced HCC in selected patients, such as surgical resection, TACE, or TACE plus RT (30-34). The National Comprehensive Cancer Network (NCCN) 

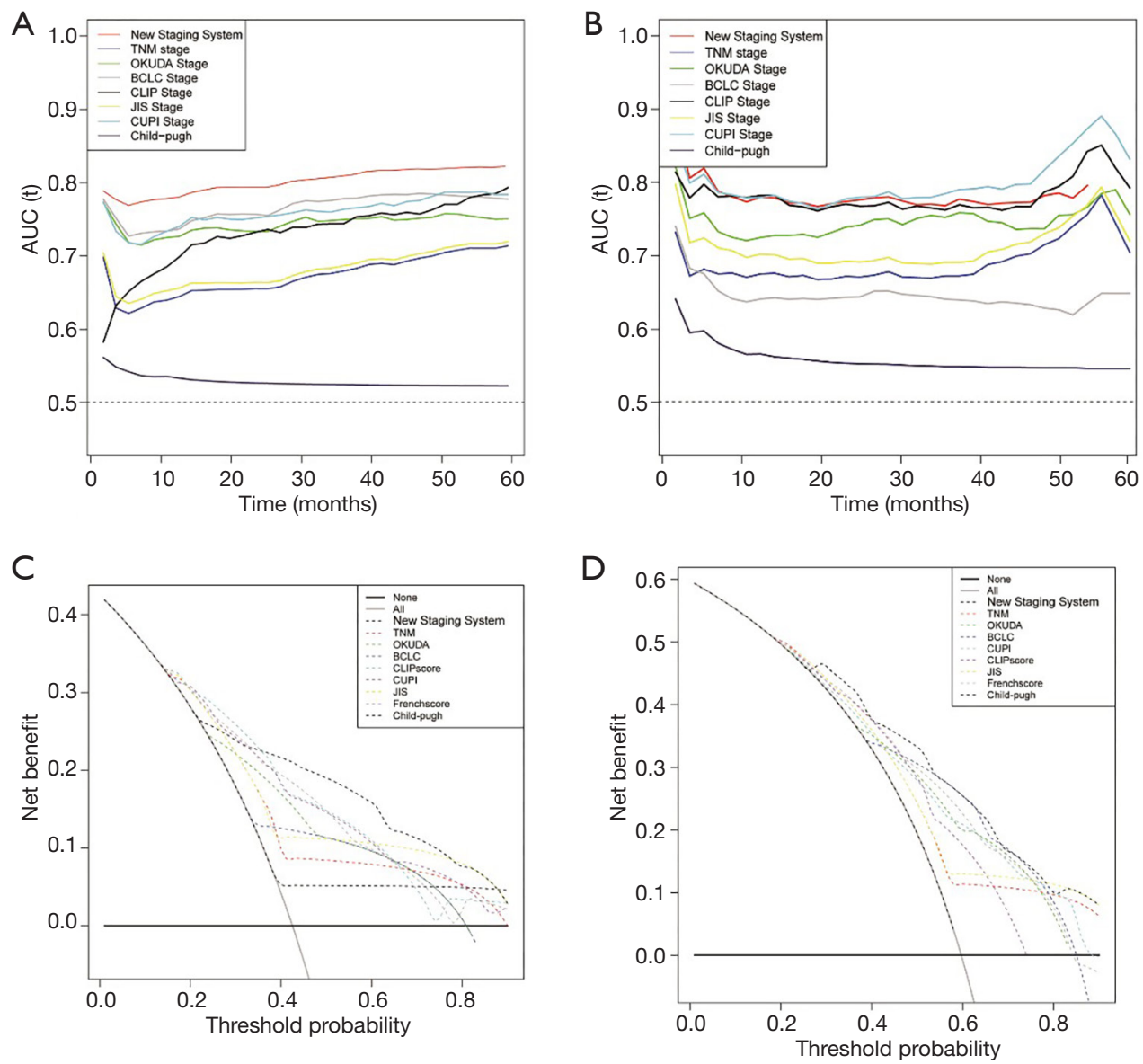

Figure 5 Comparison of the new staging system to other common standing systems. Time-dependent ROC analysis for different staging systems in the training cohort (A) and validation cohort (B); the DCA curves of different staging systems in the training cohort (C) and validation cohort (D). ROC, receiver operating characteristic curve; DCA, decision curve analysis.

Guidelines version 2.2018 for hepatocellular carcinoma suggested that hepatic resection can be considered for patients with major vascular invasion (35). A recent study with a large sample from Japan showed liver resection to result in better survival outcomes than non-surgical treatments, as long as the PVTT was confined to the first-order branch, but not involved the main portal vein (36). The median survival time in the surgery group was 1.77 years longer than the non-surgery group ( 2.87 vs. 1.10 years; $\mathrm{P}<0.001$ ), and 0.88 years longer than the non-surgery group in the propensity score-matched cohorts (2.45 vs. 1.57 years; $\mathrm{P}<0.001)$. Similar results from a large-scale, multicenter, propensity score matching analysis reported by us showed surgical treatment to be better than non-surgical treatment in patients with HCC associated with PVTT which had not involved the main portal vein and with Pugh-Child A and selected B liver function (20). A recently published metaanalysis suggested that surgical resection provided survival benefits in patients with advanced HCC when compared with TACE (37). Another recently published randomized clinical trial showed that TACE plus external radiotherapy was well-tolerated and provided good progression-free survival in patients with advanced HCC with macroscopic vascular invasion (38). An EHBH-PVTT scoring system was established as an aid to decision-making on hepatectomy for HCC patients with PVTT in our team (39). It could select appropriate HCC patients with PVTT limited to a first-order branch of the main portal vein or above for liver resection. These results indicated that careful patient selection is important in the treatment of HCC associated with PVTT. The new system refines selection of patients with HCC associated with PVTT for treatments which can 
vary from the best supportive care to liver resection.

The limitations of this study are: First, our study did not include patients with HCC associated with PVTT which coexisted with tumor thrombosis of the hepatic and inferior venous systems. Second, the majority of patients had HBVrelated HCC and all the enrolled patients came from China. Whether this new staging system can be used in patients with non-HBV-related HCC is uncertain. Third, this is a retrospective study with its inherent defects. Fourth, the grades of evidence used in this study were IIb to III only. Fifth, this study did not look into treatment mortalities, morbidities and side-effects.

In conclusion, a new staging system was proposed to provide better discriminatory ability and prognostic value for patients with HCC associated with PVTT. This system can also serve as a guide to compare the effectiveness of different treatment options for these patients.

\section{Acknowledgments}

We thank the staffs of the 5 Institutions who were involved in this study for their help in collecting the clinical data.

Funding: This work was supported by the grants of the National Science Foundation for Young Scientists of China (Grant No. 81602523); The Science Fund for Creative Research Groups (No. 81221061); the National Key Basic Research Program "973 project” (No. 2015CB554000); the China National Funds for Distinguished Young Scientists (No. 81125018).

\section{Footnote}

Reporting Checklist: The authors have completed the TRIPOD reporting checklist. Available at https://hbsn. amegroups.com/article/view/10.21037/hbsn-19-810/rc

Data Sharing Statement: Available at https://hbsn. amegroups.com/article/view/10.21037/hbsn-19-810/dss

Conflicts of Interest: All authors have completed the ICMJE uniform disclosure form (available at https://hbsn. amegroups.com/article/view/10.21037/hbsn-19-810/coif). Dr. WYL serves as an unpaid editorial board member of Hepatobiliary Surgery and Nutrition. The other authors have no conflicts of interest to declare.

Ethical Statement: The authors are accountable for all aspects of the work in ensuring that questions related to the accuracy or integrity of any part of the work are appropriately investigated and resolved. The study was conducted in accordance with the Declaration of Helsinki (as revised in 2013). The study was approved by the Ethics Committees of all the five hospitals (Permit Number: EHBHKY-2019-001-017). Informed consent was obtained from all the patients prior to treatment.

Open Access Statement: This is an Open Access article distributed in accordance with the Creative Commons Attribution-NonCommercial-NoDerivs 4.0 International License (CC BY-NC-ND 4.0), which permits the noncommercial replication and distribution of the article with the strict proviso that no changes or edits are made and the original work is properly cited (including links to both the formal publication through the relevant DOI and the license). See: https://creativecommons.org/licenses/by-nc-nd/4.0/.

\section{References}

1. IARC. Fact sheets by Population-Globocan-IARC. Available online: http://globocan. iarc.fr/Pages/fact_ sheets_population.aspx

2. Llovet JM, Bustamante J, Castells A, et al. Natural history of untreated nonsurgical hepatocellular carcinoma: rationale for the design and evaluation of therapeutic trials. Hepatology 1999;29:62-7.

3. EASL-EORTC clinical practice guidelines: management of hepatocellular carcinoma. Eur J Cancer 2012;48:599-641.

4. Lai EC, Lau WY. The continuing challenge of hepatic cancer in Asia. Surgeon 2005;3:210-5.

5. Poon RT, Fan ST, Tsang FH, et al. Locoregional therapies for hepatocellular carcinoma: a critical review from the surgeon's perspective. Ann Surg 2002;235:466-86.

6. Minagawa $M$, Makuuchi $M$. Treatment of hepatocellular carcinoma accompanied by portal vein tumor thrombus. World J Gastroenterol 2006;12:7561-7.

7. Sun JX, Shi J, Li N, et al. Portal vein tumor thrombus is a bottleneck in the treatment of hepatocellular carcinoma. Cancer Biol Med 2016;13:452-8.

8. Subramaniam S, Kelley RK, Venook AP. A review of hepatocellular carcinoma (HCC) staging systems. Chin Clin Oncol 2013;2:33.

9. Llovet JM, Bru C, Bruix J. Prognosis of hepatocellular carcinoma: the BCLC staging classification. Semin Liver Dis 1999;19:329-38.

10. EASL-EORTC clinical practice guidelines: management 
of hepatocellular carcinoma. J Hepatol 2012;56:908-43.

11. Bruix J, Sherman M, American Association for the Study of Liver D. Management of hepatocellular carcinoma: an update. Hepatology 2011;53:1020-2.

12. Bolondi L, Burroughs A, Dufour JF, et al. Heterogeneity of patients with intermediate (BCLC B) Hepatocellular Carcinoma: proposal for a subclassification to facilitate treatment decisions. Semin Liver Dis 2012;32:348-59.

13. Yau T, Yao TJ, Chan P, et al. A new prognostic score system in patients with advanced hepatocellular carcinoma not amendable to locoregional therapy: implication for patient selection in systemic therapy trials. Cancer 2008; 113:2742-51.

14. Shuqun C, Mengchao W, Han C, et al. Tumor thrombus types influence the prognosis of hepatocellular carcinoma with the tumor thrombi in the portal vein. Hepatogastroenterology 2007;54:499-502.

15. Shi J, Lai EC, Li N, et al. A new classification for hepatocellular carcinoma with portal vein tumor thrombus. J Hepatobiliary Pancreat Sci 2011;18:74-80.

16. Kudo M, Izumi N, Kokudo N, et al. Management of hepatocellular carcinoma in Japan: Consensus-Based Clinical Practice Guidelines proposed by the Japan Society of Hepatology (JSH) 2010 updated version. Dig Dis 2011;29:339-64.

17. Cheng S, Yang J, Shen F, et al. Multidisciplinary management of hepatocellular carcinoma with portal vein tumor thrombus - Eastern Hepatobiliary Surgical Hospital consensus statement. Oncotarget 2016;7:40816-29.

18. Wang K, Guo W, Li N, et al. Alpha-1-fucosidase as a prognostic indicator for hepatocellular carcinoma following hepatectomy: a large-scale, long-term study. Br J Cancer 2014;110:1811-9.

19. Adams RB, Aloia TA, Loyer E, et al. Selection for hepatic resection of colorectal liver metastases: expert consensus statement. HPB (Oxford) 2013;15:91-103.

20. Wang K, Guo WX, Chen MS, et al. Multi-modality treatment for hepatocellular carcinoma with portal vein tumor thrombus: a large scale, multicenter, propensity mathching score study. Medicine (Baltimore) 2016;95:e3015.

21. Hijazi Z, Oldgren J, Lindbäck J, et al. The novel biomarker-based ABC (age, biomarkers, clinical history)bleeding risk score for patients with atrial fibrillation: a derivation and validation study. Lancet 2016;387:2302-11.

22. Strempel S, Nendza M, Scheringer $M$, et al. Using conditional inference trees and random forests to predict the bioaccumulation potential of organic chemicals.
Environ Toxicol Chem 2013;32:1187-95.

23. Edge SB, Compton CC. The American Joint Committee on Cancer: the 7 th edition of the AJCC cancer staging manual and the future of TNM. Ann Surg Oncol 2010;17:1471-4.

24. A new prognostic system for hepatocellular carcinoma: a retrospective study of 435 patients: the Cancer of the Liver Italian Program (CLIP) investigators. Hepatology 1998;28:751-5.

25. Leung TW, Tang AM, Zee B, et al. Construction of the Chinese University Prognostic Index for hepatocellular carcinoma and comparison with the TNM staging system, the Okuda staging system, and the Cancer of the Liver Italian Program staging system: a study based on 926 patients. Cancer 2002;94:1760-9.

26. Kudo M, Chung H, Haji S, et al. Validation of a new prognostic staging system for hepatocellular carcinoma: the JIS score compared with the CLIP score. Hepatology 2004;40:1396-405.

27. Kokudo T, Hasegawa K, Matsuyama Y, et al. Liver resection for hepatocellular carcinoma associated with hepatic vein invasion: A Japanese nationwide survey. Hepatology 2017;66:510-7.

28. Cheng AL, Kang YK, Chen Z, et al. Efficacy and safety of sorafenib in patients in the Asia-Pacific region with advanced hepatocellular carcinoma: a phase III randomised, double-blind, placebo-controlled trial. Lancet Oncol 2009;10:25-34.

29. Llovet JM, Ricci S, Mazzaferro V, et al. Sorafenib in advanced hepatocellular carcinoma. N Engl J Med 2008;359:378-90.

30. Shi J, Lai EC, Li N, et al. Surgical treatment of hepatocellular carcinoma with portal vein tumor thrombus. Ann Surg Oncol 2010;17:2073-80.

31. Liu PH, Lee YH, Hsia CY, et al. Surgical resection versus transarterial chemoembolization for hepatocellular carcinoma with portal vein tumor thrombosis: a propensity score analysis. Ann Surg Oncol 2014;21:1825-33.

32. Zhou Q, Wang Y, Zhou X, et al. Prognostic analysis for treatment modalities in hepatocellular carcinomas with portal vein tumor thrombi. Asian Pac J Cancer Prev 2011;12:2847-50.

33. Ibarra RA, Rojas D, Snyder L, et al. Multicenter results of stereotactic body radiotherapy (SBRT) for non-resectable primary liver tumors. Acta Oncol 2012;51:575-83.

34. Hata M, Tokuuye K, Sugahara S, et al. Proton beam therapy for hepatocellular carcinoma with portal vein tumor thrombus. Cancer 2005;104:794-801. 
35. NCCA. Guidelines version 2.2018 for hepatocellular carcinoma. Available online: https://www.nccn.org/ professionals/physician_gls/pdf/hepatobiliary.pdf.

36. Kokudo T, Hasegawa K, Matsuyama Y, et al. Survival benefit of liver resection for hepatocellular carcinoma associated with portal vein invasion. J Hepatol 2016;65:938-43.

37. Zhang XP, Wang K, Li N, et al. Survival benefit of hepatic resection versus transarterial chemoembolization for hepatocellular carcinoma with portal vein tumor thrombus: a systematic review and meta-analysis. BMC Cancer 2017;17:902.

Cite this article as: Lau WY, Wang K, Zhang XP, Li LQ, Wen TF, Chen MS, Jia WD, Xu L, Shi J, Guo WX, Sun JX, Chen ZH, Guo L, Wei XB, Lu CD, Xue J, Zhou LP, Zheng YX, Wang M, Wu MC, Cheng SQ. A new staging system for hepatocellular carcinoma associated with portal vein tumor thrombus. HepatoBiliary Surg Nutr 2021;10(6):782-795. doi: 10.21037/hbsn-19-810
38. Yoon SM, Ryoo BY, Lee SJ, et al. Efficacy and Safety of Transarterial Chemoembolization Plus External Beam Radiotherapy vs Sorafenib in Hepatocellular Carcinoma With Macroscopic Vascular Invasion: A Randomized Clinical Trial. JAMA Oncol 2018;4:661-9.

39. Zhang XP, Gao YZ, Chen ZH, et al. An Eastern Hepatobiliary Surgery Hospital/Portal Vein Tumor Thrombus Scoring System as an Aid to Decision Making on Hepatectomy for Hepatocellular Carcinoma Patients With Portal Vein Tumor Thrombus: A Multicenter Study. Hepatology 2019;69:2076-90. 
Table S1 Patient's other characteristics of all the enrolled patients

\begin{tabular}{|c|c|c|c|c|c|c|c|c|c|c|}
\hline \multirow{2}{*}{ Variables } & \multicolumn{5}{|c|}{ Validation cohort $1(n=2,179)$} & \multicolumn{4}{|c|}{ Validation cohort $2(\mathrm{~N}=1,550)$} & \multirow[b]{2}{*}{ Stage IV $(n=82)$} \\
\hline & Stage $0(n=322)$ & Stage I $(n=745)$ & Stage II $(n=793)$ & Stage III $(n=231)$ & Stage IV $(n=88)$ & Stage $0(n=785)$ & Stage I $(n=269)$ & Stage II $(n=292)$ & Stage III $(n=122)$ & \\
\hline \multicolumn{11}{|c|}{ Gender, n (\%) } \\
\hline Male & $287(89.1)$ & $679(91.1)$ & $711(89.7)$ & $210(91.0)$ & $82(93.2)$ & $685(87.3)$ & $227(84.4)$ & $263(90.1)$ & $113(92.6)$ & $74(90.2)$ \\
\hline Female & 35 (10.9) & $66(8.9)$ & $82(10.3)$ & $21(9.0)$ & $6(6.8)$ & $100(12.7)$ & $42(15.6)$ & $29(9.9)$ & $9(7.4)$ & $8(9.8)$ \\
\hline \multicolumn{11}{|c|}{ Age, years, $n(\%)$} \\
\hline$\leq 50$ & $159(49.4)$ & $440(59.1)$ & $374(47.2)$ & $110(47.7)$ & $45(51.1)$ & $398(50.7)$ & $136(50.6)$ & $160(54.8)$ & $70(57.4)$ & $40(48.8)$ \\
\hline$>50$ & $163(50.6)$ & $305(40.9)$ & $419(52.8)$ & $121(52.3)$ & $43(48.9)$ & $387(49.3)$ & $133(49.4)$ & $132(49.2)$ & $52(42.6)$ & $42(51.2)$ \\
\hline \multicolumn{11}{|c|}{ Biochemistries, n (\%) } \\
\hline \multicolumn{11}{|c|}{ Total bilirubin (mmol/L) } \\
\hline$\leq 18.8$ & $228(70.8)$ & $543(72.9)$ & $448(56.5)$ & $130(56.3)$ & $25(28.4)$ & $603(76.8)$ & $197(73.2)$ & $179(61.3)$ & $69(56.6)$ & $26(31.7)$ \\
\hline$>18.8$ & 94 (29.2) & $202(27.1)$ & $345(43.5)$ & $101(43.7)$ & $63(71.6)$ & $182(23.2)$ & $72(26.8)$ & $113(38.7)$ & $53(43.4)$ & $56(68.3)$ \\
\hline \multicolumn{11}{|c|}{ Albumin (g/L) } \\
\hline$\leq 34$ & $10(3.1)$ & $47(6.3)$ & $154(19.4)$ & 35 (15.2) & $49(55.7)$ & $76(9.7)$ & $22(8.2)$ & 36 (12.3) & 20 (16.4) & $44(53.7)$ \\
\hline$>34$ & 312 (96.9) & $698(93.7)$ & $639(80.6)$ & $196(84.8)$ & 39 (44.3) & $709(90.3)$ & 247 (91.8) & $256(87.7)$ & $102(83.6)$ & $38(46.3)$ \\
\hline \multicolumn{11}{|l|}{ ALT $(\mu \mathrm{L} / \mathrm{L})$} \\
\hline$\leq 44$ & $185(57.5)$ & $348(46.7)$ & $350(44.1)$ & $108(46.8)$ & $40(45.5)$ & $505(64.3)$ & $164(61.0)$ & $124(42.5)$ & $58(47.5)$ & 38 (46.3) \\
\hline$>44$ & $137(42.5)$ & $397(53.3)$ & $443(55.9)$ & $123(53.2)$ & $48(54.5)$ & $280(35.7)$ & $105(39.0)$ & $168(57.5)$ & $64(52.5)$ & $44(53.7)$ \\
\hline \multicolumn{11}{|l|}{$\mathrm{HbsAg}$} \\
\hline Positive & $284(88.2)$ & $670(89.9)$ & $668(84.2)$ & $208(90.0)$ & 78 (88.7) & $682(86.9)$ & $245(91.1)$ & $262(89.7)$ & $109(89.3)$ & $72(87.8)$ \\
\hline Negative & $38(11.8)$ & 75 (10.1) & $125(15.8)$ & $23(10.0)$ & $10(11.3)$ & $103(13.1)$ & $24(8.9)$ & $30(10.3)$ & $13(10.3)$ & $10(12.2)$ \\
\hline \multicolumn{11}{|l|}{ Cirrhosis } \\
\hline Yes & $197(61.2)$ & $513(68.9)$ & $587(74.0)$ & $171(74.0)$ & $67(76.1)$ & $465(59.2)$ & $194(72.1)$ & $159(54.5)$ & $77(63.1)$ & $58(70.7)$ \\
\hline No & $125(38.8)$ & $232(31.1)$ & $206(26.0)$ & $60(26.0)$ & $21(23.9)$ & $320(40.8)$ & 75 (27.9) & $133(45.5)$ & 45 (36.9) & 24 (29.3) \\
\hline \multicolumn{11}{|c|}{ Tumor characteristics, n (\%) } \\
\hline \multicolumn{11}{|c|}{$\operatorname{AFP}(n g / m L)$} \\
\hline$\leq 400$ & $173(53.7)$ & $274(36.8)$ & $306(38.6)$ & 79 (34.2) & $20(22.7)$ & $382(48.7)$ & $110(40.9)$ & $95(32.5)$ & $44(36.1)$ & $20(24.4)$ \\
\hline$>400$ & $149(46.3)$ & $471(63.2)$ & $487(61.4)$ & $152(65.8)$ & $68(87.3)$ & $403(51.3)$ & $159(59.1)$ & $197(67.5)$ & 78 (63.9) & $62(75.6)$ \\
\hline \multicolumn{11}{|c|}{ Tumor size $(\mathrm{cm})$} \\
\hline$\leq 5$ & $149(46.3)$ & $138(18.5)$ & $119(15.0)$ & $17(7.4)$ & $6(6.8)$ & $287(36.6)$ & $62(23.0)$ & $25(8.6)$ & $9(7.4)$ & $6(7.3)$ \\
\hline$>5$ & $173(53.7)$ & $607(81.5)$ & $674(85.0)$ & $214(92.6)$ & $82(93.2)$ & $498(63.4)$ & $207(77.0)$ & $267(91.4)$ & $113(92.6)$ & $76(92.7)$ \\
\hline \multicolumn{11}{|c|}{ Tumor number } \\
\hline Single & $294(91.3)$ & $693(93.0)$ & $604(76.2)$ & $28(12.1)$ & $13(24.8)$ & $539(68.7)$ & $114(42.4)$ & $68(23.3)$ & $16(13.1)$ & $15(18.3)$ \\
\hline Multiple & $28(8.7)$ & $52(7.0)$ & $189(23.8)$ & 203 (87.9) & 75 (85.2) & 246 (31.3) & $155(57.6)$ & $224(76.7)$ & $106(86.9)$ & 67 (81.7) \\
\hline
\end{tabular}


Table S2 Median survival time of all the enrolled patients who underwent different treatments in training cohort and validation cohort

\begin{tabular}{lcc}
\hline \multirow{2}{*}{ First treatment } & \multicolumn{2}{c}{ Median survival time $(95 \% \mathrm{Cl})$} \\
\cline { 2 - 3 } Resection & Training cohort & Validation cohort \\
TACE & $17.6(15.7-19.6)$ & ${ }^{*} 35.8(34.3-37.3)$ \\
MTD or Che & $3.7(3.4-4.0)$ & $4.0(2.9-5.0)$ \\
TACE-SUR & $5.6(5.4-5.9)$ & $2.5(1.1-3.9)$ \\
RT-TACE & $8.1(7.3-9.0)$ & NA \\
TAI & $9.5(6.8-12.3)$ & NA \\
RF & NA & $7.7(6.3-9.1)$ \\
BSC & NA & $4.0(0-10.4)$ \\
\hline
\end{tabular}

*, mean survival time. CI, confidence interval; MTD, multidisciplinary therapy; Che, systemic chemotherapy; SUR, surgery; RT, external radiotherapy; TAI, transhepatic arterial infusion; RF, radiofrequency ablation; BSC, best support care.

Table S3 Patient's variables of all the nodes by the conditional inference trees in training cohort

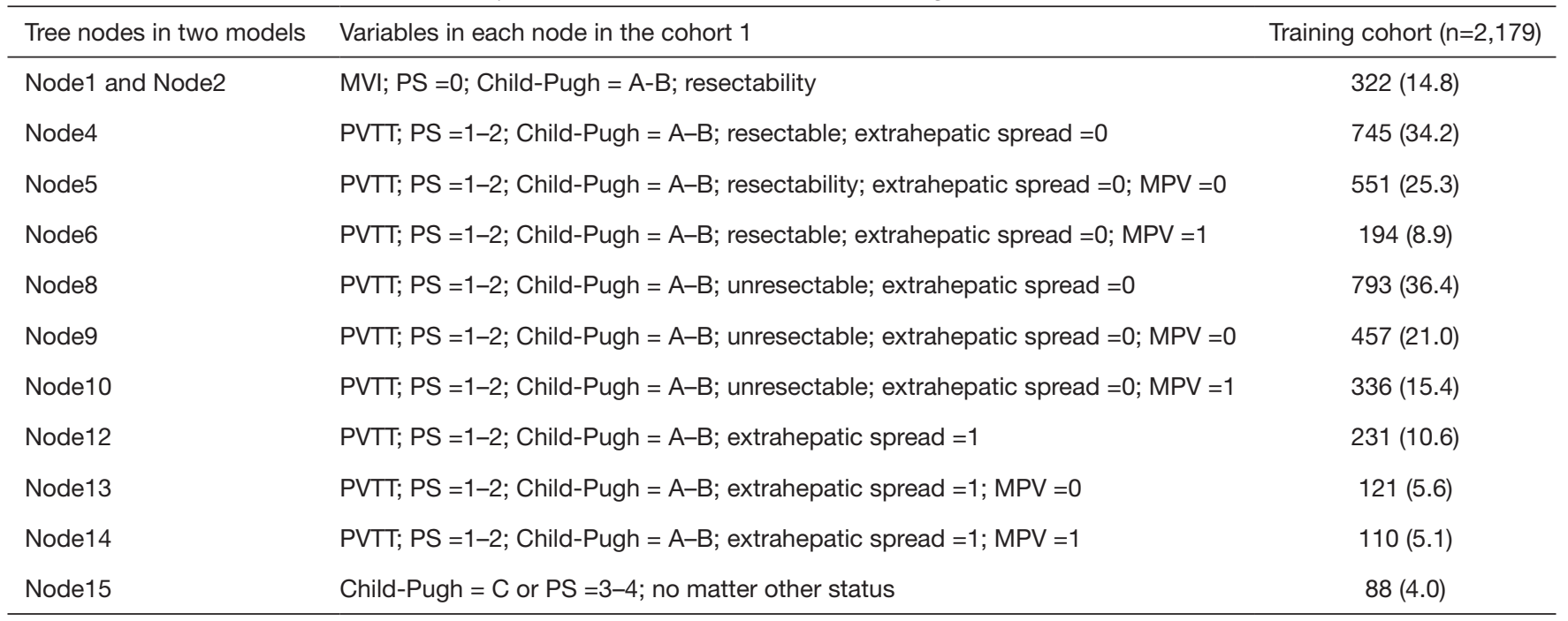

Table S4 The scaled Schoenfeld residual plots

\begin{tabular}{lcccc}
\hline & & & Relative coefficient \\
\cline { 2 - 4 } & 0 & 1 & 2 & 3 \\
\hline Child-Pugh & A-B & & C \\
PVTT & No & & $1-2$ & $3-4$ \\
ECOG -PS & 0 & Yes & \\
Extrahepatic Metastasis & No & Yes & \\
Tumor resectability & No & Yes & \\
MPV & No &
\end{tabular}

MPV, main portal vein. 
Table S5 The 1-, 2- and 3-year overall and median survival outcomes of all the enrolled patients in the new staging system

\begin{tabular}{|c|c|c|c|c|c|c|c|c|}
\hline & \multicolumn{2}{|c|}{ 1-year } & \multicolumn{2}{|c|}{ 2-year } & \multicolumn{2}{|c|}{ 3-year } & \multicolumn{2}{|c|}{ Median survival time $(95 \% \mathrm{Cl})$} \\
\hline & Training cohort & Validation cohort & Training cohort & Validation cohort & Training cohort & Validation cohort & Training cohort & Validation cohort \\
\hline \multicolumn{9}{|l|}{ Stage } \\
\hline 0 & 86.3 & 78.1 & 69.7 & 67.5 & 61.9 & 61.4 & $57.1(37.2-76.9)$ & *37.6 (35.9-39.2) \\
\hline I & 50.2 & 65.6 & 28.9 & 56.2 & 19.3 & 48.6 & $12.1(11.0-13.2)$ & *30.4 (27.4-33.4) \\
\hline II & 26.8 & 29.6 & 8.7 & 12.8 & 2.9 & 12.8 & $5.7(5.1-6.2)$ & $6.4(4.9-7.9)$ \\
\hline III & 10.6 & 12.5 & NA & & & & $4.0(3.3-4.6)$ & $2.8(1.3-4.4)$ \\
\hline IV & 0 & 1.2 & NA & & & & $2.5(1.7-3.3)$ & $1.5(1.3-1.7)$ \\
\hline \multicolumn{9}{|c|}{ Sub-stage } \\
\hline IA & 55.5 & 67.3 & 33.2 & 59.3 & 23.1 & 50.9 & $13.9(12.1-15.7)$ & *31.5 (28.4-34.7) \\
\hline IB & 35.1 & 55.4 & 16.9 & 33.3 & 8.3 & 33.3 & $6.0(4.4-7.7)$ & $15.0(8.3-21.7)$ \\
\hline$\| A$ & 29.7 & 34.8 & 11.8 & 17.8 & 3.8 & 17.8 & $6.4(5.6-7.2)$ & $6.4(4.5-8.3)$ \\
\hline IIB & 20.8 & 22 & 4.7 & 5.6 & 1.7 & NA & $4.0(3.0-4.9)$ & $5.7(3.2-8.3)$ \\
\hline IIIA & 10.4 & 16.1 & NA & & & & $3.4(2.1-4.6)$ & $4.5(2.6-6.4)$ \\
\hline IIIB & 0 & 6.7 & NA & & & & $3.3(2.6-4.0)$ & $1.8(1.2-2.4)$ \\
\hline
\end{tabular}

${ }^{*}$, mean survival time. $\mathrm{Cl}$, confidence interval. 